\title{
Cost reduction of a hybrid energy storage system considering correlation between wind and PV power
}

\author{
Lin Feng $^{1 *}$, Jingning Zhang ${ }^{1}$, Guojie Li ${ }^{1}$ and Bangling Zhang ${ }^{2}$
}

\begin{abstract}
A hybrid energy storage system (HESS) plays an important role in balancing the cost with the performance in terms of stabilizing the fluctuant power of wind farms and photovoltaic (PV) stations. To further bring down the cost and actually implement the dispatchability of wind/PV plants, there is a need to penetrate into the major factors that contribute to the cost of the any HESS. This paper first discusses hybrid energy storage systems, as well as chemical properties in different medium, deeming the ramp rate as one of the determinants that must be observed in the cost calculation. Then, a mathematical tool, Copula, is explained in details for the purpose of unscrambling the dependences between the power of wind and PV plants. To lower the cost, the basic rule for allocation of buffered power is also put forward, with the minimum energy capacities of the battery ESS(BESS) and the supercapacitor ESS(SC-ESS) simultaneously determined by integration. And the paper introduces the probability method to analyze how power and energy is compensated in certain confidence level. After that, two definitions of coefficients are set up, separately describing energy storage status and wind curtailment level. Finally, the paper gives a numerical example stemmed from real data acquired in wind farms and PV stations in Belgium. The conclusion presents that the cost of a hybrid energy storage system is greatly affected by ramp-rate and dependence between the power of wind farms and photovoltaic stations, in which dependence can easily be determined by Copulas.
\end{abstract}

Keywords: Probability analysis, Copula, Correlation, Capacity optimization, Hybrid ESS

\section{Introduction}

Owing to the fact of the fast development of renewable energy and the increased concern of the environmental and sustainable impact of fossil fuels, wind farms and photovoltaic plants have been widely increasingly built around the world over past decades [1]. Wind farms and photovoltaic plants are currently not confined in the conventional off-grid generation systems. Those on-grid variable power sources, however, exert an adverse impact on the operation and control of conventional power grid [2]. The power of wind farms and PV plants is normally much more dependent upon the on-site landform, topography and climate. As a result, they inherently contain the congenital defects, including intermittency, fluctuation and undispatchablility. Hence, energy storage

\footnotetext{
* Correspondence: fenglin@sjtu.edu.cn

${ }^{1}$ School of Electrical Information and Electronic Engineering, Shanghai Jiao

Tong University, Shanghai, China

Full list of author information is available at the end of the article
}

systems which nowadays have become less expensive are introduced in such systems to ease the instability tendency of grids, with the ability of compensating for intermitted and fluctuant outputs of wind/PV plants. As the cost gradually falls, energy storage systems have been put into use in grids on a larger scale. Single type energy storage systems cannot meet the demands in real applications, considering the power and energy requirement at different time scale. As a result, hybrid energy storage systems turn out to be the feasible choice. The most common hybrid energy storage system is composed of batteries, such as advanced lead-acid or lithium-ion batteries, and supercapacitors [3].

Refs. $[4,5]$ present a fundamental frame to analyze the cost in a hybrid energy storage system, pointing out the ramp rate is the principal factor that has effect on the cost. Copula functions are discussed in [6, 7]. The spacetime complementarities between wind farms and PV stations are emphasized in [8]. 
Ref. [9] cautiously explored the wind curtailment phenomenon in nations partly driven by wind power generation, such as America, Spain and Denmark. A new wind-ESS combined control method for surpassing Wind curtailment are in detail discussed in [10].

To further bring down the cost and actually implement the dispatchability of wind/PV plants, hybrid energy storage systems will be increasingly pervasive in the foreseeable future. Therefore, there is a need to penetrate into the major factors that contribute to the cost of the any HESS.

In this study, based on the cost of HESS by considering the ramp rate mentioned in [4], Copulas functions are proposed to analyze the dependence between wind and PV power. Then the cost of HESS is analyzed considering the ramp rate as well as the dependence between wind and PV power. Simulation studies are carried out to verify the above analysis.

\section{Hybrid energy storage system}

A hybrid energy storage system often owns the merits of individual single energy storage system. A battery energy storage system, such as advanced lead-acid batteries, has the advantages in pricing and large-scale using. It, however, poorly performs in the situation where power soars dramatically, or on the contrary, drops instantly. So, it is supposed to accumulate massive less fluctuant energy. With the more costly price, a supercapacitor energy storage system is not equipped with possibility of extensive using, but it does well in the moments when power moves fast.

The Fig. 1 depicts the diagram of a common fundamental generation system with a HESS, in which power equipment are omitted. The arrows point out the direction of power in an instant.

In Fig. 1, $P_{\mathrm{v}}$ represents the output power of photovoltaic stations. $P_{\mathrm{w}}$ represents the power of wind farms. $P$ is the aggregated power of $P_{\mathrm{v}}$ and $\mathrm{Pw}$. Buffered power is represented by $P_{\mathrm{h}}$. $P_{\mathrm{sc}}$ is the power of the supercapacitor energy storage system. $P_{\mathrm{b}}$ is the power of the battery energy storage system. $P_{\mathrm{d}}$ represents the dispatched power into the grid.

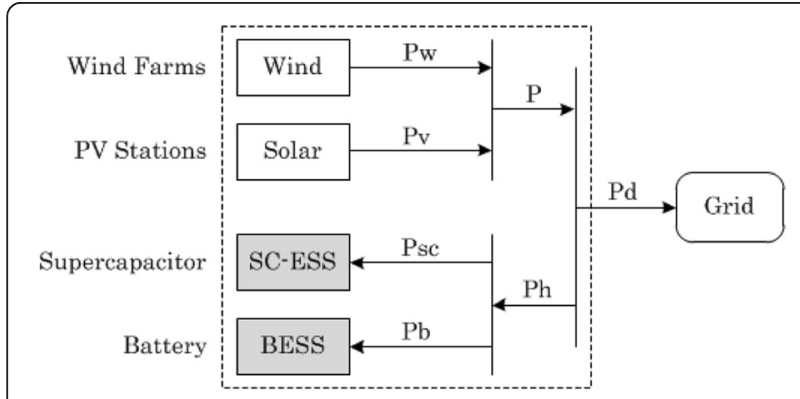

Fig. 1 The diagram of a HESS-PV/Wind System

\section{Ramp rate}

The ramp rate represents the change rate of power. More specifically, ramp rates, including ramp-up rates and rate-down rates, can be approached in two respects. First, they cannot be separated from wind farms or PV stations. For not only protecting wind turbines, photovoltaic cells and girds, but also ensuring that the power fluctuation of wind farms and PV plants can be regulated by other conventional power sources, like fossil-fueled plants, there is a limit to maximum change rate of active power in the wind farms and photovoltaic stations. Tables 1 and 2 bring the standards in China.

Second, ramp rates also go for the energy storage system. Owing to the chemical properties and specifications by battery manufacturers, a battery energy storage system has the boundaries of maximum ramp rate as well. There is no limit to ramp rates in the supercapacitor energy storage system, which undoubtedly assumes the superiority of supercapacitor energy storage systems.

\section{Method}

By Copulas, the dependences, or correlations between random variables can be set up. On one hand, marginal distributions can easily be obtained with joint probability distribution. On the other hand, it is not easy to get the joint distribution when marginal distributions are known. The emergence and improvement of Copulas functions, to some extent, resolves the problem [6].

\section{Definition}

In 1959, Sklar put forward the thinking of Copula function, which divides N-dimension joint probability distribution into $\mathrm{N}$ marginal distribution functions and one copula function that describes the correlation degree between variables. Nelsen gave the strict definition of the Copula function in 1999. Copula, denoted as $C\left(u_{1}, u_{2}, \ldots, u_{\mathrm{N}}\right)$, is a connection function that links joint probability distribution $F\left(X_{1}, X_{2}, \ldots, X_{\mathrm{N}}\right)$ of random variables $X_{1}, X_{2}, \ldots, X_{\mathrm{N}}$ with their individual marginal distribution, $F_{\mathrm{X} 1}\left(x_{1}\right), \ldots, F_{\mathrm{XN}}\left(x_{\mathrm{N}}\right)$, which can be explicitly formulated as follows.

Table 1 The limitation of ramp-rates in wind farms

\begin{tabular}{lll}
\hline Installed capacity & $\begin{array}{l}\text { Maximum variation } \\
\text { in } 10 \mathrm{~min}(\mathrm{MW})\end{array}$ & $\begin{array}{l}\text { Maximum variation } \\
\text { in } 1 \mathrm{~min}(\mathrm{MW})\end{array}$ \\
\hline$<30$ & 20 & 6 \\
$30-150$ & capacity/1.5 & capacity/5 \\
$>150$ & 100 & 30 \\
\hline
\end{tabular}

Technical rule for wind power plant connected to Power Grid in 2009 
Table 2 The limitation of ramp-rates in PV stations

\begin{tabular}{lll}
\hline Project scale & $\begin{array}{l}\text { Maximum variation } \\
\text { in } 10 \mathrm{~min}(\mathrm{MW})\end{array}$ & $\begin{array}{l}\text { Maximum variation } \\
\text { in } 1 \mathrm{~min}(\mathrm{MW})\end{array}$ \\
\hline small & capacity & 0.2 \\
medium & capacity & capacity $/ 5$ \\
large & capacity/3 & capacity/10 \\
\hline
\end{tabular}

Technical rule for photovoltaic power station connected to Power Grid in 2011

$$
F\left(X_{1}, X_{2}, \ldots, X_{N}\right)=C\left[F_{X_{1}}\left(x_{1}\right), F_{X_{2}}\left(x_{2}\right) \ldots F_{X_{N}}\left(x_{N}\right)\right]
$$

\section{Common copulas}

Supposing Pearson coefficient is, a two-dimensional Gaussian Copula function is as follows,

$$
\begin{gathered}
C(u, v ; \rho)=\int_{-\infty}^{\Phi^{-1}(u)} \int_{-\infty}^{\Phi^{-1}(v)} \frac{1}{2 \pi \sqrt{1-\rho^{2}}} \exp \\
{\left[-\frac{s^{2}-2 \rho s t+t^{2}}{2\left(1-\rho^{2}\right)}\right] d s t d t}
\end{gathered}
$$

A two-dimensional $\mathrm{t}$-Copula function with free degree $\mathrm{k}$ is as follows,

$$
\begin{aligned}
C(u, v ; \rho, k)= & \int_{-\infty}^{t_{k}^{-1}(u)} \int_{-\infty}^{t_{k}{ }^{-1}(v)} \frac{1}{2 \pi \sqrt{1-\rho^{2}}} \\
& {\left[1+\frac{s^{2}-2 \rho s t+t^{2}}{k\left(1-\rho^{2}\right)}\right] d s d t }
\end{aligned}
$$

Archimedean copulas are defined as,

$$
\begin{aligned}
C\left(u_{1}, u_{2}, \ldots, u_{N}\right)=\left\{\begin{array}{l}
\phi^{-1}\left[\phi\left(u_{1}\right), \phi\left(u_{2}\right), \ldots, \phi\left(u_{N}\right)\right] \\
0
\end{array}\right. & \sum_{i=1}^{N} \phi\left(u_{1}\right) \leq \phi(0) \\
& \text { other }
\end{aligned}
$$

\section{The measurement of correlation}

There are several measurements of correlation, or dependency, between random variables. With regard to different usage, massive of them can be adopted, such as Pearson coefficient $\rho$, Kendall rank correlation coefficient $\tau$, Spearman rank correlation coefficient $\rho$ and tail dependence $\lambda$.

If marginal distributions of continual random variable $(X, Y)$ are $F(x)$ and $G(y)$, the relationships between Copula function $C(u, v)$ and Kendall rank correlation coefficient $\tau$, Spearman rank correlation coefficient $\rho$, tail dependence $\lambda$ are as follows, respectively.

$$
\begin{aligned}
& \tau=4 \int_{0}^{1} \int_{0}^{1} C(u, v) d C(u, v)-1 \\
& \rho_{s}=12 \int_{0}^{1} \int_{0}^{1} C(u, v) d u d v-3 \\
& \lambda^{u p}=\lim _{u \rightarrow 1^{-}} \frac{1-2 u+C(u, v)}{1-u} \\
& \lambda^{l o}=\lim _{u \rightarrow 0^{+}} \frac{C(u, v)}{u}
\end{aligned}
$$

\section{Results}

\section{Dispatchability}

Generally, a generation plant bids against each other. It is common for bidders in several power trading markets to forecast its power (nowadays, more and more forecast data are purchased from third-party service providers) and to propose generation schedule $N$ hours before the market begins every trading day, which can be exemplified with Nordic power exchange market. The mechanism is called $N$-hour rule. To simulate the operation of real world, a forecast example is given below, with the hypothesis that dispatched power is derived from hourly average of forecasting power. A forecast algorithm using historical power data can be found in Fig. 2.

\section{The allocation of $P_{\mathrm{b}}$ and $P_{\mathrm{sc}}$}

$P_{\mathrm{h}}$ is the aggregated power of $P_{\mathrm{b}}$ and $P_{\mathrm{sc}} \cdot P_{\mathrm{h}}$ is the power that buffers from/into hybrid energy storage system. $Y$ is the ramp rate. $P_{\mathrm{b}}$ and $P_{\mathrm{sc}}$ are confirmed in (9) and (10) [4].

Supposing

$$
\begin{aligned}
& P_{B, r}\left(t_{i-1}\right)+Y \cdot \Delta t \geq P_{H, r}\left(t_{i}\right) \\
& P_{B, r}\left(t_{i}\right)=P_{H, r}\left(t_{i}\right), P_{S C, r}\left(t_{i}\right)=0
\end{aligned}
$$

Otherwise,

$$
\begin{aligned}
& P_{B, r}\left(t_{i}\right)=P_{B, r}\left(t_{i-1}\right)+Y \cdot \Delta t \\
& P_{S C, r}\left(t_{i}\right)=P_{H, r}\left(t_{i}\right)-P_{B, r}\left(t_{i}\right)
\end{aligned}
$$

\section{The determination of energy capacity}

After integrating $P_{\mathrm{b}}$ and $P_{\mathrm{sc}}$ with time, $E_{\mathrm{b}}$ and $E_{\mathrm{sc}}$ can be achieved. $E_{\mathrm{b}}$ and $E_{\mathrm{sc}}$ display the energy variation, or the energy level, of the energy storage system [4]. In Fig. 3, the zero-interface represents the assumptive initial energy level. The energy storage system is charged when the curve goes up with system being discharged as the curve comes down. Thus, the minimum energy capacity of the BESS or the SC-ESS is based on its own moving 


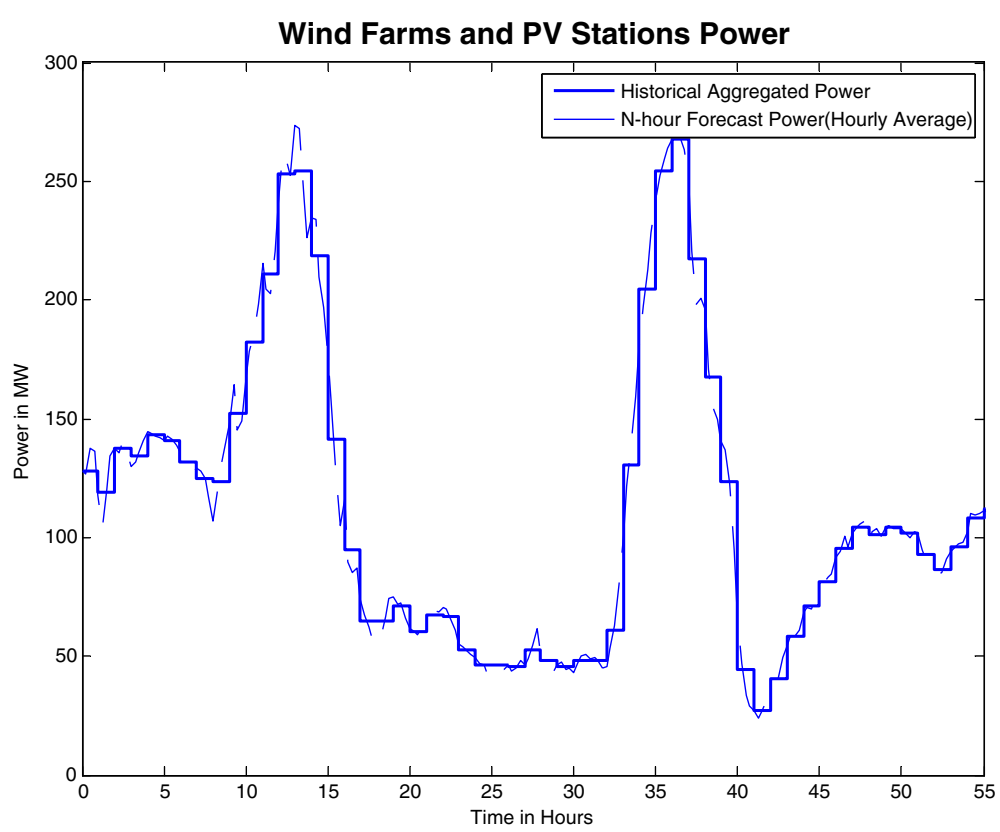

Fig. 2 An example of forecast power

range, respectively. This implies that the best energy capacity is no less than these least value.

\section{Cost function}

Solving (9) and (10), every ramp rate $Y$ corresponds to a set of $P_{\mathrm{b}}(t)$ and $P_{\mathrm{sc}}(t)$, from which $E_{\mathrm{b}}(t)$ and $E_{\mathrm{sc}}(t)$ can be gained. The fit value of $P_{\mathrm{b}}$ is the maximum of $P_{\mathrm{b}}(t)$; the same for $P_{\mathrm{sc}}$. Note that the fit values of $E_{\mathrm{b}}$ and $E_{\mathrm{sc}}$ depend primarily on their difference between upper and lower bounds in Fig. 3.

Then, the basic cost function is as follows [4].

$$
f=k_{1} \times E_{b}+k_{2} \times E_{S C}+k_{3} \times P_{b}+k_{3} \times P_{S C}
$$

where $k_{1}, k_{2}, k_{3}$ and $k_{4}$ are obtained in [11].

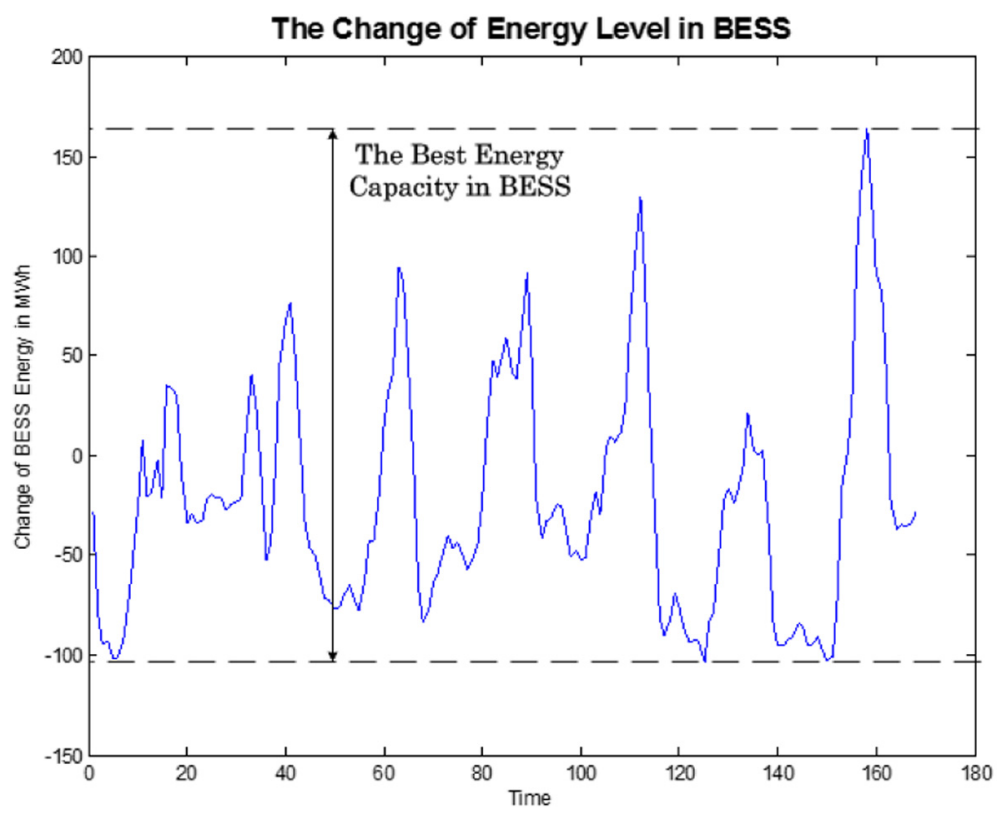

Fig. 3 The determination of the BESS energy capacity 


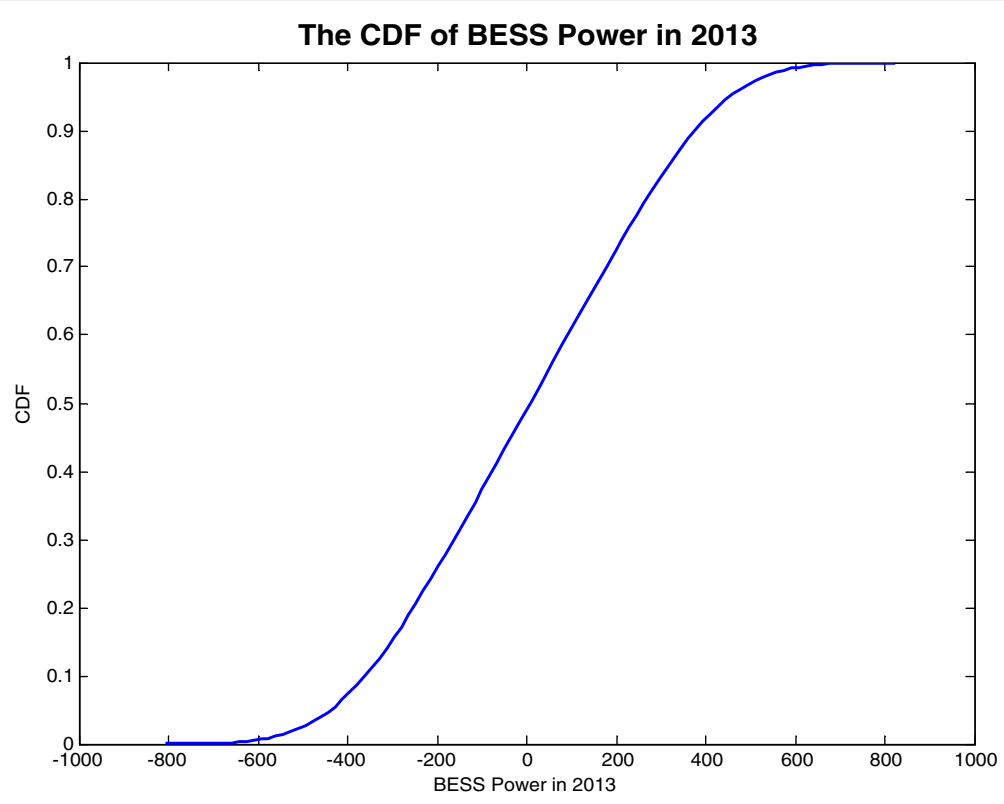

Fig. 4 The CDF of BESS Power in 2013

\section{Statistical observation}

To minimize the cost $f$, the proper ramp rate $Y$ should be selected. Meanwhile, $P_{b}(\mathrm{t}), P_{s c}(\mathrm{t}), E_{b}(\mathrm{t})$ and $E_{s c}(\mathrm{t})$ are successively determined. It is natural that the Cumulative Distribution Function (CDF) of these capacities is obtained as shown in Fig. 4 to depict the complete spectacle in statistical manners [4].

\section{Definitions of coefficients}

To make the problem more intuitive, here we separately define two types of coefficients, wind curtailment coefficient and energy storage coefficient. This approach will undoubtedly construct the connection between energy storage status and wind curtailment condition. The similar solar power abandonment is ignored for parallel approach.

The key to the problem is that we make a probabilistic modification to wind power output. In other words, $P_{w}$ is replaced by $\left[1-\xi\left(\mathrm{t}_{\mathrm{i}}\right)\right] \cdot P_{w}^{\prime}$; note that $P_{w}^{\prime}$ is the original $100 \%$ of wind power.

Now we choose $\xi\left(\mathrm{t}_{\mathrm{i}}\right)$ to describe the level of wind curtailment and the definition of $\xi\left(\mathrm{t}_{\mathrm{i}}\right)$ is as follows,

$$
\xi\left(\mathrm{t}_{\mathrm{i}}\right)=\left\{\begin{array}{l}
0, \quad \text { zerowind curtailment } \\
0 \sim 1, \quad \text { partlywind curtailment } \\
1, \quad \text { complete wind curtailment }
\end{array}\right.
$$

Then, the wind curtailment coefficient is denoted by,

$$
C=\frac{\sum_{i}^{i+k} \xi\left(\mathrm{t}_{\mathrm{i}}\right)}{T / \Delta t} \times 100 \%
$$

Finally, the energy storage compensation coefficient is represented,

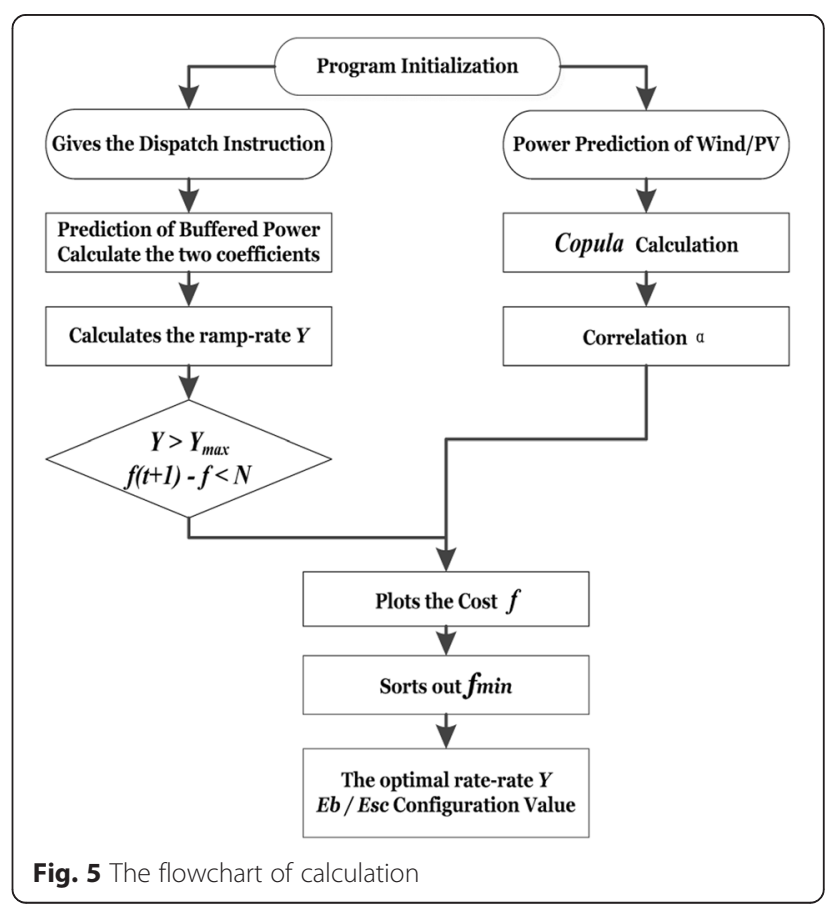




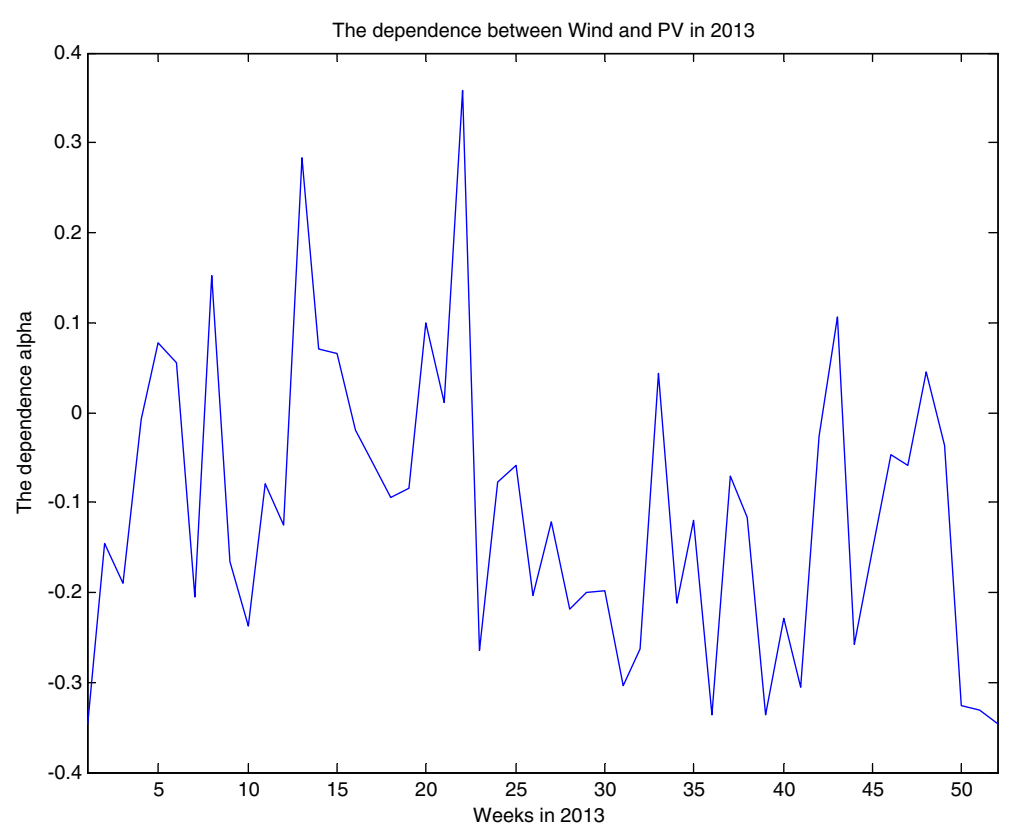

Fig. 6 The dependence between wind and PV in 2013

$$
\lambda=\frac{\sum_{i}^{i+k}\left|\left[1-\xi\left(\mathrm{t}_{\mathrm{i}}\right)\right] P_{w}+P_{\nu}-P_{H, r}\right| \cdot \Delta t}{\sum_{i}^{i+k} P_{h} \cdot \Delta t}
$$

As a result, we obtain that

- $\lambda>1$, the cost of hybrid energy storage system goes up

- $\lambda=1$, the cost of hybrid energy storage system remains unchanged

- $\lambda<1$, the cost of hybrid energy storage system goes down

\section{Procedure to determine the optimum BESS ramp-rates}

The MATLAB program is developed following the methods mentioned above. The flowchart is shown in Fig. 5.

\section{Discussion}

Here, the paper exhibits a numerical example. All the data are based on real historical scene. The installed capacities of wind farms and PV stations are 143.45 MW and 431.17 MW, respectively, which are located at Belgium (http:// www.elia.be).

\section{The dependence using copulas}

The paper adopts Copulas to readily calculate the dependences between wind farms and photovoltaic stations, which are shown in Fig. 6.
From the above dependences, three typical sets of data, representing three different common dependences, are picked up. The copula PDF of one of the three sets of data is given in Fig. 7.

\section{The buffered power}

The paper utilizes the method in [12] to forecast the dispatchable power $P_{\mathrm{d}}$ into the grid in next $N$ hours. Note that the following procedures are based merely on one set of data. Then, $P_{\mathrm{h}}$ is obtained after $P$ deducting $P_{\mathrm{d}}$. An example of $P_{\mathrm{h}}$ is shown in Fig. 8 .

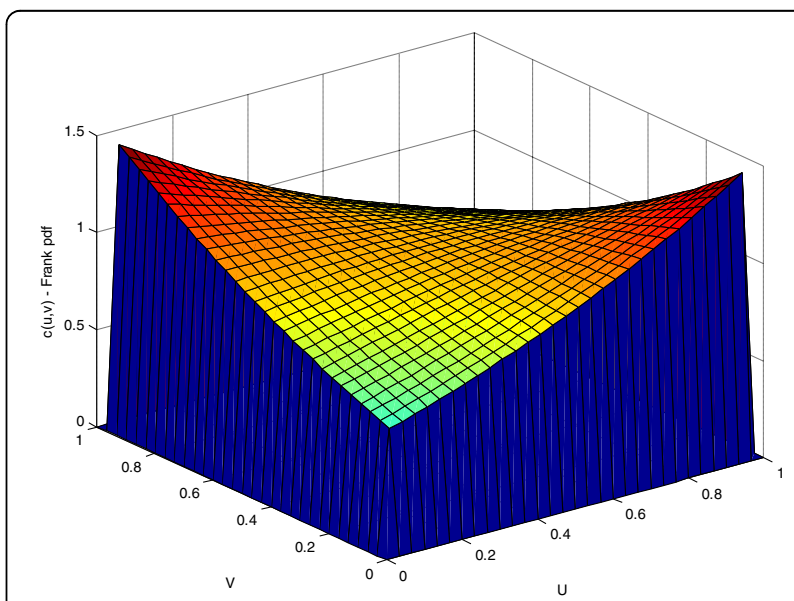

Fig. 7 The PDF of Frank Copula between wind/PV 


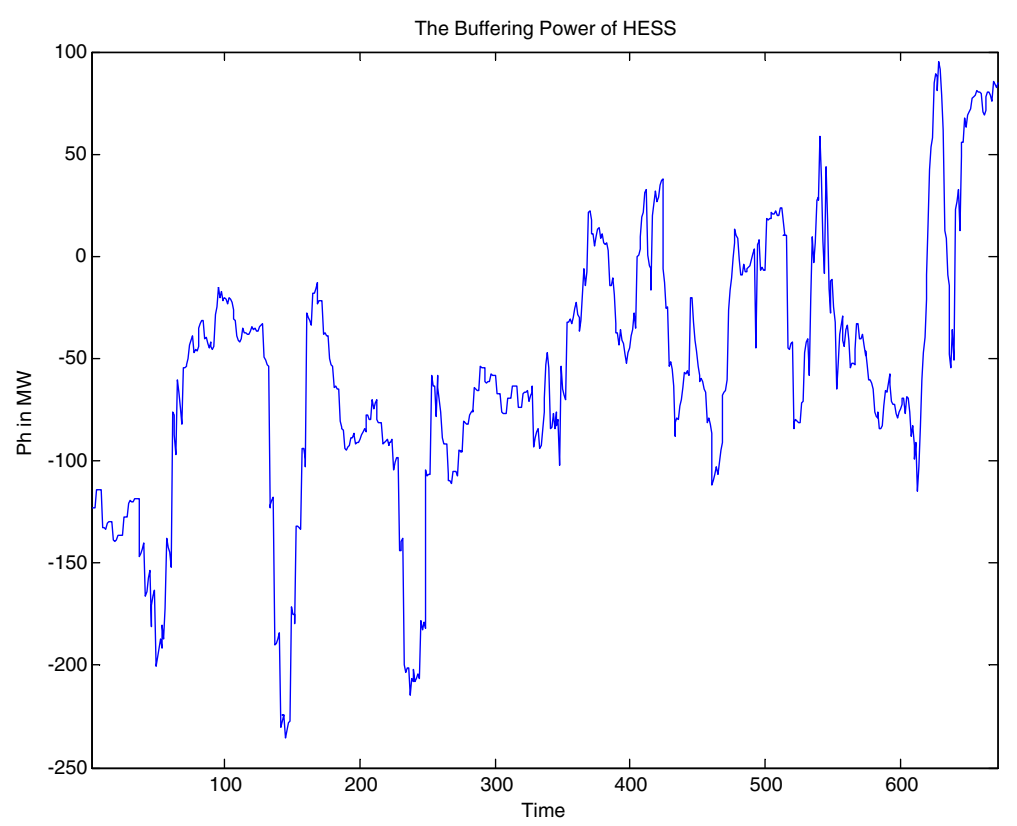

Fig. 8 An example of buffered power $P_{\mathrm{h}}$

\section{Power and energy capacities}

Once ramp rate $Y$ is decided, a set of corresponding data is determined as well, including $P_{\mathrm{b}}, P_{\mathrm{sc}}, E_{\mathrm{b}}$ and $E_{\mathrm{sc}}$, where energy level $E_{\mathrm{b}}$ and $E_{\mathrm{sc}}$ are from the integral of output powers. Here, the designed MATLAB Program endeavors to traverse $Y$ from 0 through 10 . Then, a great number of sets of $\left\{P_{\mathrm{b}}, P_{\mathrm{sc}}, E_{\mathrm{b}}, E_{\mathrm{sc}}\right\}$ are acquired by the method mentioned in Subsection Cost function. The allocation of $P_{\mathrm{b}}$ and $P_{\mathrm{sc}}$ is revealed in Fig. 9. It is not difficult to find out that majority of $P_{\mathrm{h}}$ is supported by the BESS, while the minority is assumed by SC-ESS in this case.

The essence of cost function

As seen in (11), the cost of the HESS can be obtained by traversing ramp rate $Y$. By the input of three sets of data,

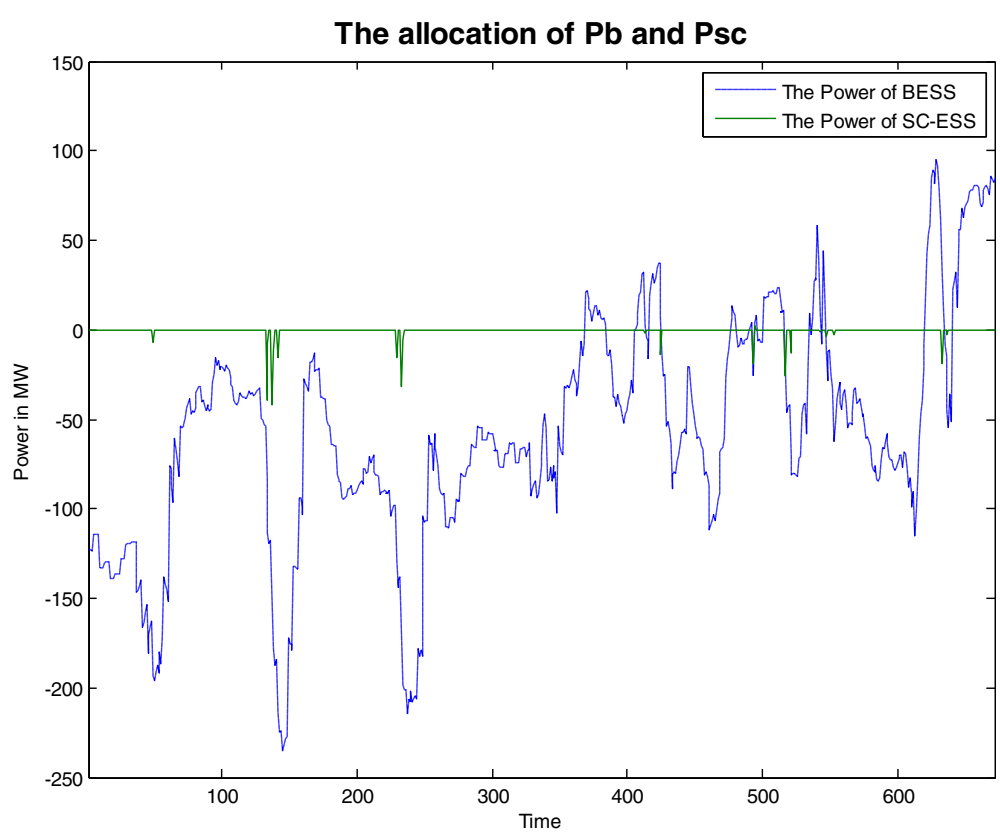

Fig. 9 An allocation of $P_{\mathrm{b}}$ and $P_{\mathrm{sc}}$ 


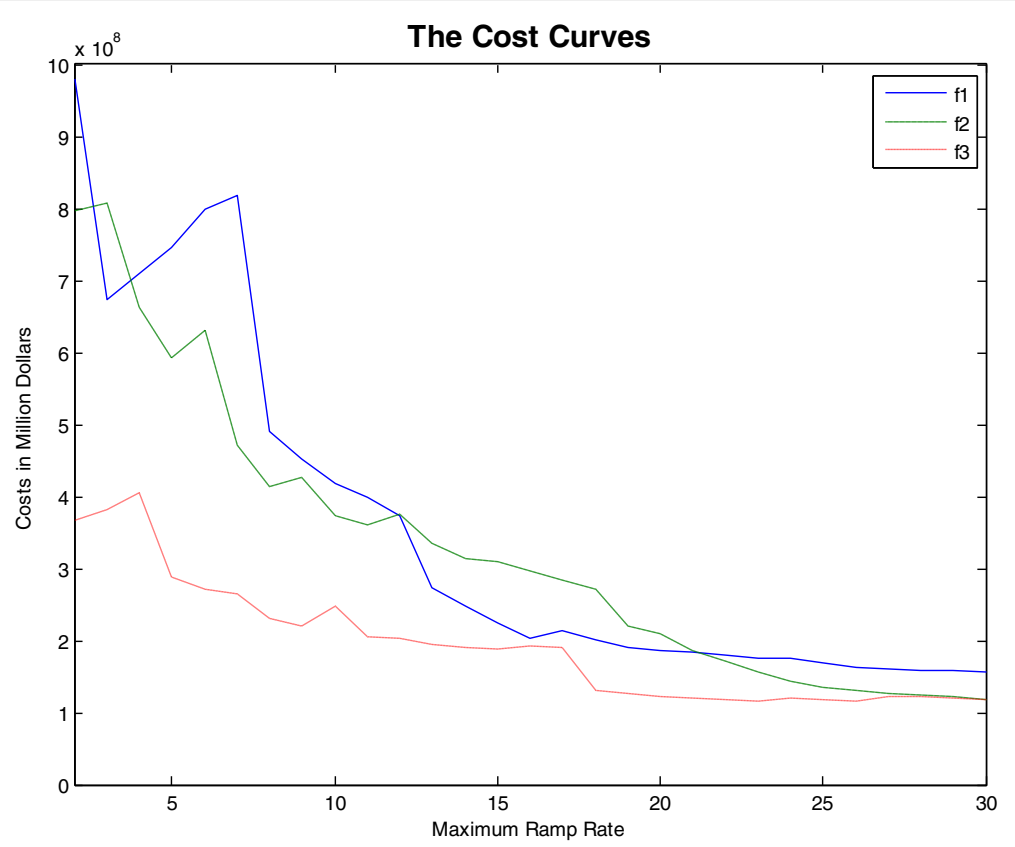

Fig. 10 The cost curves with different dependences

the Fig. 10 intuitively demonstrates the relation between cost and dependence.

Hence, the essence of the HESS cost is as follows.

$$
f=f(Y, \alpha, \lambda ; t)
$$

In (12), $f_{1}, f_{2}$ and $f_{3}$ correspond the dependence $-0.1,-0.2$ and -0.3 , respectively. As the dependence $\alpha$ between wind farms and PV stations goes up, the cost $f$ diminishes. On determining the best ramp rate $Y$, the minimum energy capacities of the BESS and the SC-ESS are also confirmed. The cost curves with different lambda are showed in Fig. 11.

\section{Conclusion}

The paper concluded that the cost of a hybrid energy storage system is greatly affected by ramp-rate and

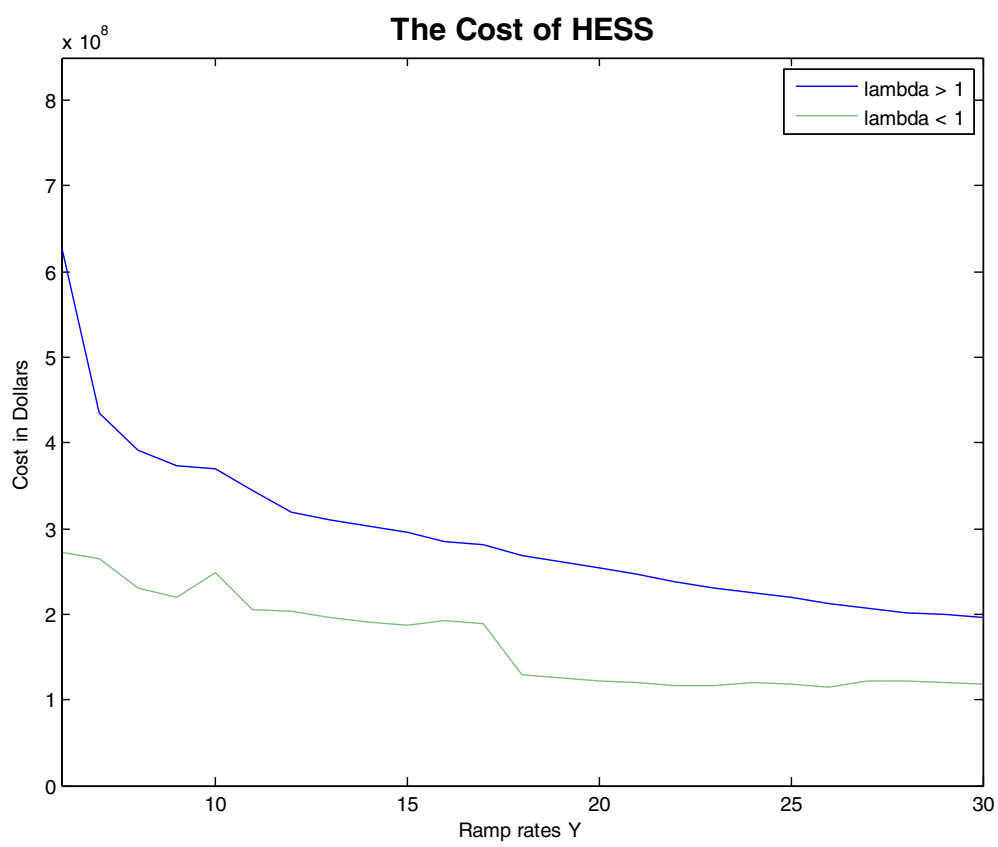

Fig. 11 The cost curves with different lambda 
dependence between the power of wind farms and photovoltaic stations, in which dependence can easily be determined by Copulas. A numerical example shows as the dependence between wind farms and PV stations goes up, the cost decreases. Moreover, the cost is also influenced by energy storage compensation coefficient.

\section{Acknowledgements}

This work was supported by Shanghai Science and Technology Committee (13231204002) and National Key Technology R\&D Program of China (2015BAA01B02).

\section{Authors' contribution}

LF and JNZ carried out the theoretic studies, calculated the numerical example and drafted the manuscript; GJL and BLZ participated in the theoretic studies. All authors read and approved the final manuscript.

\section{Competing interests}

The authors declare that they have no competing interests.

\section{About the Authors}

L. Feng She is now a lecturer in the Dept. of Electrical Engineering, Shanghai Jiao Tong Univ., Shanghai, China. Her research interests are the control and the integration of renewable energy, and Microgrid.

J. N. Zhang He is currently pursuing the Master Degree in SEIEE from Shanghai Jiao Tong University. His research interests are capacity optimization and energy storage systems.

G. J. Li He is now a professor in the Dept. of Electrical Engineering, Shanghai Jiao Tong Univ., Shanghai, China. His current research interests include power system analysis and control, wind and PV power control and integration, and Microgrid.

B. L. Zhang He is currently working for Shanghai Power \& Energy Storage Battery System Engineering Tech. Co. Ltd.. His research interests are the electric power system design and the integration of new energy resources.

\section{Author details}

${ }^{1}$ School of Electrical Information and Electronic Engineering, Shanghai Jiao Tong University, Shanghai, China. ${ }^{2}$ Shanghai Power \& Energy Storage Battery System Engineering Tech. Co. Ltd., Shanghai, China.

Received: 13 May 2016 Accepted: 16 May 2016

Published online: 06 July 2016

\section{References}

1. Ashwin Kumar, A. (2010). A study on renewable energy resources in India. In International Conference on Environmental Engineering and Applications (ICEEA) (pp. 49-53).

2. MacGill, I. F. (2012). Impacts and best practices of large-scale wind power integration into electricity markets -Some Australian perspectives (Power and Energy Society General Meeting, pp. 1-6)

3. Dai, H. (2010). A Study on Lead Acid Battery and Ultracapacitor Hybrid Energy Storage System for Hybrid City Bus (Optoelectronics and Image Processing (ICOIP), 2010 International Conference on. IEEE, Vol. 1, pp. 154-159).

4. Wee, K. W., Choi, S. S., \& Vilathgamuwa, D. M. (2011). Design of a renewable-hybrid energy storage power scheme for short-term power dispatch (Electric Utility Deregulation and Restructuring and Power Technologies (DRPT), 2011 4th International Conference on. IEEE, pp. 1511-1516)

5. Yao, D. L., Choi, S. S., \& Tseng, K. J. (2011). Design of short-term dispatch strategy to maximize income of a wind power-energy storage generating station. In Innovative Smart Grid Technologies Asia (ISGT) (pp. 1-8)

6. Papaefthymiou, G. (2009). Using Copulas for Modeling Stochastic Dependence in Power System Uncertainty Analysis. IEEE Transactions on Power Systems, 24(1), 40-49.

7. Brunel, N. J.-B. (2010). Modeling and Unsupervised Classification of Multivariate Hidden Markov Chains With Copulas. IEEE Transactions on Automatic Control, 55(2), 338-349.
8. Zhang, N., Kang, C., Xu, Q., Jiang, C., Chen, Z., \& Liu, J. (2013). Modelling and Simulating the Spatio-Temporal Correlations of Clustered Wind Power Using Copula. Journal of Electrical Engineering and Technology, 8(6), 1615-1625.

9. Wang, Q.-k. (2012). Update and Empirical Analysis of Domestic and Foreign Wind Energy Curtailment. East China Electric Power, 40(3), 378-381.

10. Zhang, N., Kang, C., Xu, Q., Jiang, C., Chen, Z., \& Liu, J. (2013). Wind-ESS Combined Control for Suppressing Ramp Rates of Wind Power. Automation of Electric Power Systems, 37(13), 17-23.

11. Chen, H., Cong, T. N., Yang, W., Tan, C., Li, Y., \& Ding, Y. (2009). Progressin Energy Storage System: a Critical Review. Progress in Natural Science, 19(3), 291-312.

12. Mahoney, W. P., Parks, K., Wiener, G., Liu, Y., Myers, W. L., \& Sun, J. (2012) A wind power forecasting system to optimize grid integration. IEEE Transactions on Sustainable Energy, 3(4), 670-682.

\section{Submit your manuscript to a SpringerOpen ${ }^{\circ}$ journal and benefit from:}

- Convenient online submission

- Rigorous peer review

- Immediate publication on acceptance

- Open access: articles freely available online

- High visibility within the field

- Retaining the copyright to your article

Submit your next manuscript at $>$ springeropen.com 